\title{
Prekäre Freiheit - Die Arbeit von Kreativen
}

\author{
Alexandra Manske \\ Janet Merkel
}

Die Ausweitung von prekären Arbeits- und Beschäftigungsformen gilt spätestens seit Robert Castels Diagnose zur Metamorphose der sozialen Frage als ein strukturelles Merkmal des Wandels der Arbeit. Dass sich auch im sogenannten „Kreativsektor“ prekäre Arbeitsund Lebensformen ausweiten, bleibt meist unterbelichtet. Dieser Beitrag lenkt die Aufmerksamkeit auf die Arbeit von Kreativen und damit auf ein expandierendes Arbeitsmarktsegment, das insbesondere für (groß)städtisch geprägte Arbeitsmärkte an Bedeutung gewinnt. Die These ist, dass die Erwerbsbedingungen in der Kreativwirtschaft zu einer Normalisierung und Verstetigung von Prekarität im Sinne von rechtlich und finanziell unsicheren Beschäftigungsformen beitragen. ${ }^{1}$

\section{1 \\ Problemaufriss}

Kultur und Kreativität gelten als neue Schlüsselressourcen im Aufbau wettbewerbsfähiger und wissensbasierter Dienstleistungsökonomien. So ist im Abschlussbericht der Enquete-Kommission „Kultur in Deutschland“ nachzulesen: „Die Kulturwirtschaft hat sich zu einer Zukunftsbranche mit Wachstums- und Beschäftigungspotenzial entwickelt. Hier entstehen Arbeitsplätze und Wertschöpfung - vor allem in Klein- und Kleinstunternehmen. Sie sind das Kraftzentrum der Kulturwirtschaft und der Beginn der Wertschöpfungskette" (Kultur in Deutschland 2007, S. 7). Dieses „Kraftzentrum“ der Kultur- und Kreativwirtschaft, die stetig anwachsende Gruppe der Alleinunternehmer (Selbstständige ohne Beschäftigte), steht im Fokus des folgenden Beitrages.

Wie lässt sich die steigende Anzahl von Alleinunternehmern in der Kulturwirtschaft beurteilen? Wie ist ihre soziale Lage einzuschätzen? Handelt es sich hier etwa um die Ausweitung atypischer und/oder prekärer Arbeitsverhältnisse? Diese Schlussfolgerung liegt angesichts der vielfach radikal marktvermittelten Arbeit in der Kreativwirtschaft durchaus nahe. Sie setzt aber voraus, die Arbeits- und Erwerbsbedingungen der Kreativwirtschaft vor dem Hintergrund der die fordistische Phase prägenden Strukturen von Arbeit, Betrieb und Beschäftigung zu analysieren und das Normalarbeitsverhältnis als Kontrastfolie heranzuziehen. Ein solcher Ansatz ist im vorliegenden Fall problematisch, weil die Erwerbsarbeit von Künstlern und Kreativen traditionell häufig weder der lohnabhängigen noch der selbstständig unternehme- rischen Tätigkeit entspricht. So sind prekäre Arbeitsbedingungen ebenso ein typisches Merkmal von Künstlerarbeitsmärkten wie ihre arbeitspolitische Regulierung von jeher schwächer ist als in fordistisch geprägten Beschäftigungsverhältnissen und Wirtschaftssektoren (Haak/Schmid 1999).

Insofern lautet die These, dass die Arbeitsverhältnisse in der Kreativwirtschaft nur bedingt in der Weise als „atypisch“ oder „prekär“ beschrieben werden können, wie der Begriff gegenwärtig in der sozialwissenschaftlichen Prekaritätsdebatte gefasst wird, nämlich als Beschäftigungsverhältnisse, die soziale Regulationen und Schutzmechanismen des fordistischen Arbeitsmodells unterlaufen (Mayer-Ahuja 2003, S. 14; Dörre 2005).

In der Kreativwirtschaft, so wird hier argumentiert, handelt es sich demgegenüber um eine Prekarisierung auf hohem Niveau (Manske 2007): Eine hohe Bildung trifft mit einer starken künstlerisch-kreativen Motivation zusammen und vermischt sich mit wirtschaftlichen Armutsrisiken und subjektiven Autonomiegewinnen. Obgleich in diesem Muster eine gewisse historische Kontinuität angelegt ist, weisen sowohl die strukturelle Expansion der Kreativwirtschaft, ihre politische Indienstnahme als auch die subjektiven Verarbeitungsmuster der spezifischen Erwerbsbedingungen in der Kreativwirtschaft darauf hin, dass Kreative für die Ausweitung instabiler beruflicher Existenzen stehen und daher deutlich machen, wie herkömmliche Kriterien von sozialer Ungleichheit brüchig und traditionelle Stratifikationsmuster der Bundesrepublik infrage gestellt werden. Welche Handlungsstrategien kreative Alleinunternehmer im Arbeitsalltag entwickeln, um sich dennoch subjektiv befriedigende Handlungsspielräume zu erhalten, wird im Folgenden vorgestellt.
Empirische Basis der Ausführungen sind drei qualitative Untersuchungen. Sie beruhen auf insgesamt 48 problemzentrierten Akteursinterviews, durchgeführt im Zeitraum von 2001 bis 2007, einer Vielzahl informeller Gespräche und ethnografischer Feldbeobachtungen sowie auf Expertengesprächen mit Vertretern aus Politik, Verwaltung und Wirtschaft zur Situation kreativwirtschaftlicher Unternehmen in Berlin. Die Ausführungen rekurrieren im engeren Sinn auf eine Auswahl des Datenmaterials aus dem Jahr 2007. Insofern handelt es sich zwar nicht um eine Paneluntersuchung, jedoch bauen die Studien aufeinander auf, ergänzen sich und stellen eine perspektivische Erweiterung dar, da es sich erstens um eine Untersuchung zu Alleinunternehmern in der IT-Wirtschaft (Manske 2007), zweitens um eine Studie

\footnotetext{
1 Für konstruktive Kritik an einer früheren Version dieses Artikels danken wir G. Günter Voß sowie zwei anonymen Gutachtern bzw. Gutachterinnen.
}

Alexandra Manske, Dr., Soziologin und Politikwissenschaftlerin, wissenschaftliche Mitarbeiterin an der Humboldt Universität zu Berlin, Institut für Sozialwissenschaften Soziologie der Arbeit und Geschlechterverhältnisse. Arbeitsschwerpunkte: Wandel der Arbeitsgesellschaft und ihrer Ungleichheitskonfigurationen, Urbane Kreativmilieus, Geschlechterforschung e-mail alexandra.manske@tu-berlin.de Janet Merkel, Sozialwissenschaftlerin, wissenschaftliche Mitarbeiterin am Wissenschaftszentrum Berlin für Sozialforschung (WZB) in der Abteilung "Kulturelle Quellen von Neuheit". Arbeitsschwerpunkte: Stadtsoziologie, Kreativwirtschaft e-mail: jmerkel@wzb.eu 
über künstlerisch-gestaltende Berufe (Merkel 2009) und drittens um eine Kurzstudie zu Geisteswissenschaftlern in der Kreativwirtschaft (Manske/Merkel 2008²) handelt.

Die von uns befragten Akteure der Kreativwirtschaft sind alle zwischen 1970 und 1975 geboren. Damit gehören sie jener Kohorte von Kreativen an, die in den 1990er Jahren in dieses Wirtschaftssegment eingestiegen ist und damit in der Phase sich verschärfender Arbeitsmarktbedingungen ihren beruflichen Werdegang begonnen hat (Gottschall 1999). Ihre Tätigkeiten lassen sich den Wirtschaftszweigen Musik, Darstellende Kunst, Film/Radio/TV sowie dem IT-Bereich zuordnen.

Im Folgenden führen wir zunächst in die Diskussion um Kreativwirtschaft ein und legen dar, aus welcher Perspektive die Handlungsspielräume im Arbeitsalltag kreativer Alleinunternehmer beobachtet werden, um dann im Anschluss unsere empirischen Befunde zu präsentieren.

\section{2 \\ Kreativwirtschaft in der Diskussion}

Die Kreativwirtschaft ist ein anwachsendes Wirtschafts- und Arbeitsfeld. Laut Enquete-Bericht „Kultur in Deutschland“ lag der Anteil der Kreativwirtschaft an der Bruttowertschöpfung der Bundesrepublik im Jahr 2004 mit 36 Mrd. $€$ zwischen dem der Chemischen Industrie und der Energiewirtschaft. Die Gesamtzahl der Erwerbstätigen im Kultursektor betrug bundesweit im Jahr 2003 rund 800.000 Personen und ist seit 1995 um rund $33 \%$ angewachsen (Kultur in Deutschland 2007, S. 336f.).

Diese wirtschaftlichen Daten sind aus sozialwissenschaftlicher Perspektive weniger eindeutig. Je nachdem, ob man den wirtschaftpolitischen Begriff „Kreativwirtschaft" zugrunde legt oder den analytischen „Kulturberufe“, operiert man mit einem weiten Begriff von Kreativwirtschaft oder einem engen von Kulturdienstleistungen. Unter Kreativwirtschaft werden die bislang als „Kulturwirtschaft" bezeichneten Wirtschaftszweige Musik, Film, Verlagswesen, Architektur, Design und darstellende Kunst sowie die „Kreativbranchen“ Software/Multimedia und Werbung gefasst (Söndermann 2007, S. 9). „Kulturdienstleistungen“" hingegen beschreiben ein Spektrum von Berufsfeldern, das künstlerisch- kreative, publizistische und kulturvermittelnde Berufe erfasst (Schnell 2008).

Der vorliegende Beitrag knüpft an beide Untersuchungsrichtungen an und verortet sich an ihrer Schnittstelle, indem er die weite Definition von Kreativwirtschaft verwendet, aber einen arbeitsmarkttheoretischen und ungleichheitstheoretischen Zugang zum Thema wählt. Dabei spiegeln die folgenden Untersuchungsergebnisse insofern ein für Berlin typisches Phänomen wider, als in Berlin Honorare niedriger, die Unternehmen weniger umsatzstark sind und bundesweit die höchste Konzentration an Soloselbstständigen zu finden ist. Die strukturellen Rahmenbedingungen für Alleinunternehmer sind jedoch dieselben wie andernorts, sodass sie Rückschlüsse auf die arbeitsmarktpolitischen Gegebenheiten in der Kreativwirtschaft insgesamt zulassen.

\subsection{KREATIVWIRTSCHAFT IN DER ERWERBS- UND ARBEITSSOZIOLOGIE}

Erwerbsverhältnisse in der Kreativwirtschaft werden seit Ende der 1990er Jahre mit einem starken Fokus auf Kulturdienstleistungen arbeitssoziologisch betrachtet (Betzelt 2006; Gottschall 1999; Haak/ Schmid 1999). So liegen Untersuchungen vor, die etwa die Geschlechterarrangements von Paaren in den alten und neuen Medien, d.h. im Print- und Online-Journalismus (Henninger 2004), oder anhand der sozialen Lage von IT-Alleinunternehmern neue Prekarisierungskonfigurationen ins Licht rücken (Manske 2007).

„Creative Industries" hingegen ist ein Begriff aus der Wirtschaftspolitik und hat seinen Ursprung in den 1990er Jahren in Großbritannien. Die damalige New Labour Regierung wollte die steigende wirtschaftliche Bedeutung der Bereiche Kunst und Kultur hervorheben und sich von dem bis dato gebräuchlichem Konzept der „Cultural Industries“, der damit verbunden Orientierung auf Kulturpolitik und den öffentlich gesponserten Kultursektor absetzen. Die britische Regierung definiert "Creative Industries" als "those activities which have their origin in individual creativity, skill and talent and which have the potential for wealth and job creation through the generation and exploitation of intellectual property" (Creative Industries Mapping Document 2001, S. 5).

Die Begriffe „Kreativwirtschaft“ und „Kulturdienstleistungen“ beschreiben somit unterschiedliche empirische Sachver- halte mit spezifisch analytischem Fokus. Wir beziehen uns auf Akteure in der Kreativwirtschaft, die ebenso Kulturdienstleister umfasst wie die in der weiten Definition erfassten Akteursgruppen.

Die Untersuchungsgruppe besteht folglich nicht aus klassischen Künstlern, sondern aus selbstständigen Kulturdienstleistern (Betzelt 2006), „, neuen Kulturunternehmern“ (Mandel 2007) und „Culturepreneurs“ (Lange 2007). Ihr hervorstechendes Merkmal ist, dass sie an den Schnittstellen klassischer Kultursektoren (öffentlich, gemeinnützig, privatwirtschaftlich) neue Tätigkeitsprofile und Marktnischen entwickeln. Ein spezifisches Kennzeichen von Erwerbsarbeit in der Kreativwirtschaft ist demnach, dass die für den Bereich der Kulturdienstleistungen übliche Differenzierung zwischen primären und sekundären Kulturberufen verschwimmt (Betzelt 2006, S. 6). Denn Akteure der Kreativwirtschaft sind ebenso künstlerisch tätig, wie sie marktbezogen mit erwerbswirtschaftlichem Zweck agieren.

Unsere Befunde zeigen, dass sich das Arbeitshandeln von Kreativen gerade durch eine Verknüpfung der beiden Differenzierungsmerkmale auszeichnet. Indem die Akteure „primäre“ mit „sekundären“ Merkmalen zu einem spezifischen Arbeitshandeln verzahnen, wirken sie aktiv an der Konstituierung neuer Erwerbsfelder und Berufsprofile mit. So schöpfen Kreative gewissermaßen ihr eigenes Berufsprofil und bilden einen Individualberuf aus, der sich nach G. Günter Voß durch eine „reflexive Verberuflichung“ und eine „relativierte Fachlichkeit“ auszeichnet. Die sozialen Eigenschaften solcher „Individualberufler“ seien von einer konjunktur-, branchenund lebensabhängigen Dynamik geprägt, die im hohen Maße durch Kontingenz gezeichnet sei (Voß 2007, S. 107).

\footnotetext{
2 Die Untersuchung beleuchtet die Kreativwirtschaft als Zusammenspiel von politischer Inszenierung und wirtschaftlicher Dynamik. Sie wurde vom Bundesministerium für Bildung und Forschung (BMBF) finanziert. In dem Untersuchungsbericht finden sich auch Angaben zum theoretischen Zugriff sowie zu methodischer Verfahrensweise und Kategorien. Die empirischen Befunde zeigen zwei voneinander zu differenzierende Handlungsstrategien: der "Feste Freie" in arbeitnehmerähnlicher Erwerbssituation und der "Selbstunternehmer" in selbstständiger Erwerbsform (Manske/Merkel 2008). Beide Muster sind nicht neu, doch weiten sich in der Kulturwirtschaft nicht regulierte Erwerbsformen seit den 1980er Jahren überproportional aus (Gottschall 1999).
} 


\subsection{WER SIND DIE „KREATIVEN“?}

Kreative Alleinunternehmer und ihre wirtschaftliche Situation zu beschreiben, ist aufgrund der statistischen Datenlage bislang nur unzureichend möglich. Auch der Mikrozensus, die Beschäftigtenstatistik sowie das Soziooekonomische Panel (SOEP) bieten nur wenig verlässliche Daten über die sozialstrukturelle Lage kreativwirtschaftlicher Akteure; zudem stellt sich deren Auswertung und Interpretation als problematisch dar (vgl. zum Datenproblem Betzelt 2006; Manske 2007). Die statistischen Daten müssen daher durch qualitative Erhebungen ergänzt und gestützt werden.

Knapp 80 \% der Unternehmen der Kultur- und Kreativwirtschaft sind kleinteilig organisiert, d.h. sie haben im Durchschnitt weniger als fünf Angestellte. Es wird jedoch vermutet, dass die Mehrzahl durch Einzelunternehmer getragen wird (Kultur in Deutschland 2007, S. 339; Söndermann 2007, S. 16). Für die kreativwirtschaftlichen Branchen in Berlin kommt eine Erhebung des Deutschen Instituts für Wirtschaftsforschung zu dem Ergebnis, dass ca. $45 \%$ aller Beschäftigten als Freiberufler und Soloselbstständige tätig sind (Geppert/ Mundelius 2007). Obwohl die Angaben für verschiedene Branchen und Städte schwanken, ist der Selbstständigenanteil in der Kreativwirtschaft deutlich höher als in der Gesamtwirtschaft (Schulze Buschoff 2006).

Die verfügbaren Daten weisen zudem auf ein überdurchschnittlich hohes Bildungsniveau der Kreativen hin (Betzelt 2006). Mit Blick auf die Prekaritätsdebatte zeigt sich folglich eindeutig, dass „prekär“ nicht automatisch "schlecht qualifiziert" und „sozial marginalisiert“ heißt (Leicht/ Philipp 2005).

Die Einkommen von „Kreativen“ sind schwankend und polarisiert. Laut Mikrozensus unterschreitet mindestens ein Fünftel der kreativen Alleinunternehmer die derzeitige Armutsrisikogrenze von $938 €$ netto monatlich. Anderseits zeigen die Daten auch, dass ein großer Anteil der kreativen Alleinunternehmer in materieller Hinsicht nicht unter prekären Bedingungen lebt (Kultur in Deutschland 2007, S. 290). Insgesamt sind die Einkommensbedingungen in der Kreativwirtschaft äußerst disparat. Allerdings lässt sich die soziale Lage der „Kreativen“ auf Basis der Einkommensteuerstatistik oder des Mikrozensus nur unzureichend darstellen (ebd., S. 291).
Abzugrenzen ist die Untersuchungsgruppe auch von Richard Floridas Konzept der kreativen Klasse. Floridas Ausführungen sind von der Diskussion um die Kreativwirtschaft zu unterscheiden, da er verschiedene höherqualifizierte Berufsgruppen untersucht, die er als „kreativ“ bezeichnet, aber nicht die Kreativwirtschaft im Besonderen (Florida 2002, S. 68f.). Vielmehr sind Kultur und Kreativität in seiner Argumentation attraktive Standortfaktoren, um wissensintensive Industrien und Dienstleistungen in Städten anzusiedeln.

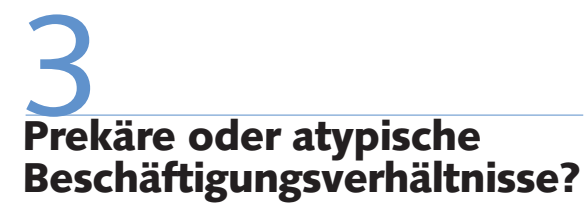

Nicht alle Beschäftigungsformen, die vom Standard des Normalarbeitsverhältnisses abweichen, sind notwendigerweise prekär. Das verdeutlichen beispielsweise die „freien Berufe" (Anwälte, Ärzte etc.) oder das Beamtentum. Ob ein Beschäftigungsverhältnis prekär oder atypisch ist, hängt außerdem vom Beobachtungsstandpunkt ab. In den 1980er Jahren wurden vom Normalarbeitsverhältnis abweichende Beschäftigungsverhältnisse häufig als atypisch bezeichnet; dies galt vor allem für frauenspezifische Beschäftigungen (Mayer-Ahuja 2003). Nach Keller/Seifert (2006) stellen prekäre Beschäftigungsverhältnisse eine Unterkategorie von atypischer Beschäftigung dar, sofern es sich um die Umwandlung von Normalarbeitsverhältnissen in gering abgesicherte Beschäftigungsverhältnisse handelt. Dieser Unterscheidung entsprechend wird Prekarität in der Regel anhand von drei Strukturdimensionen gemessen:

- materiell-reproduktive Dimension: prekär sei Erwerbsarbeit dann, wenn sie als Haupteinnahmequelle nicht existenzsichernd ist und/oder eine Tätigkeit unsicher, da zeitlich befristet ist;

- institutionell-rechtliche Dimension: prekär sei Erwerbsarbeit dann, wenn sie von tariflichen Schutzbestimmungen ausgeschlossen ist und keinen ausreichenden sozialen Schutz vor den Wechselfällen des Lebens sowie im Alter bietet;

- sozial-kommunikative Dimension: prekär sei Erwerbsarbeit dann, wenn sie nicht gleichberechtigt in soziale Netze am Ar- beitsort integriert ist (Dörre 2005, S. 181; Mayer-Ahuja 2003, S. 14).

Aus Gründen der Vergleichbarkeit werden die empirischen Befunde zur Situation von Kreativen am Berliner Arbeitsmarkt entlang der drei Prekaritäts-Dimensionen diskutiert. Hierfür werden kurz die strukturellen Rahmenbedingungen für die Kreativwirtschaft sowie die Handlungsstrategien der befragten Kreativen im alltäglichen Umgang mit dem Prekarisierungspotenzial ihres Individualberufs erläutert. Dabei wird sich zeigen, dass die Abstufung von „atypischer" versus „prekärer" Beschäftigung für das Untersuchungsfeld nur bedingt trägt, weil die Umwandlung von Beschäftigungsverhältnissen nur einen Ausschnitt des Problems reflektiert. Zudem kommt die Spezifik der untersuchten Soziallage in ihrer Tiefenschärfe erst zum Ausdruck, wenn auch die subjektiven Verarbeitungsformen untersucht werden. Sie werden entlang der jeweiligen strukturellen Dimension dargestellt, was eine methodische Verschränkung zwischen struktureller und subjektiver Ebene gewährleistet.

\subsection{PREKARITÄT IM ALLTAG KREATIVER ALLEINUNTERNEHMER}

\section{MATERIELL-REPRODUKTIVE DIMENSION}

Die Expansion des Arbeitsmarktes Kreativwirtschaft geht mit einer Polarisierung innerhalb unseres Untersuchungsfeldes zuungunsten der Mikro- und Alleinunternehmer einher (Kultur in Deutschland 2007, S. 336ff.). Die materielle Lage der Alleinunternehmer ist in der Regel prekär und bestätigt statistische Befunde aus Untersuchungen zur sozialen Lage von Künstlern (Haak 2005). Die Einkommen der Befragten umfassen nach Selbstauskünften eine Spanne von $10.000 €$ bis $29.000 €$ pro Jahr und liegen damit weit unter dem Durchschnittsverdienst von Akademikern und nah an der Armutsrisikogrenze. Die starke Streuung ist typisch für den Kreativbereich, das geringe Einkommen ist typisch für Alleinselbstständige. Dabei zeigt sich ein weiteres Muster aus anderen Kulturbereichen: Die Kombination von überdurchschnittlicher Bildung mit unterdurchschnittlichem Einkommen. Beschäftigungsgruppen aus dem Kreativ- und Kulturbereich stehen offenbar für eine 
strukturelle Entkoppelung der meritokratischen Triade von Bildung - Beruf - Einkommen und verdeutlichen, wie sich industriegesellschaftliche Statusbedingungen in bestimmten Zonen des sozialen Raums auflösen (Betzelt 2006; Haak 2005; Manske 2007).

Da meist auf Rechnung gearbeitet wird und Honorare erst (lange Zeit) nach Fertigstellung der Projekte gezahlt werden, müssen die Akteure üblicherweise in Vorleistung treten. Die langen Entwicklungsund Vorbereitungsphasen, sich überlagernde Projekte sowie die erzwungenen Freiräume zwischen den Projekten, in denen kein Anschlussprojekt vorhanden ist, und, wie ein Befragter es ausdrückt, die „chronische Unterfinanzierung, bei der man alle Risiken allein trägt", strukturieren die wirtschaftliche Lage der Befragten und machen das Leben zu einem schwierigen und unberechenbaren "Abenteuer“. Die materielle Ungewissheit und die starken Schwankungen empfinden die Befragten teilweise als „brutal“, weil sie oft nicht wissen, wie sie ihren Lebensunterhalt bestreiten sollen.

Als besonders kritisch empfinden die Befragten ihre soziale Lage, wenn sie auf Stipendien bzw. Engagements in öffentlichen Kultureinrichtungen angewiesen sind. Die langen Vorlaufzeiten zur Projektbewilligung erfordern eine vorausschauende Planung und entsprechende Rücklagen, um diese Phase finanziell zu überbrücken. Wie prekär eine solche Existenz werden kann, zeigt das Beispiel einer befragten $\mathrm{Ku}-$ ratorin, die zwar in der Berliner Off-Kultur-Szene als erfolgreich gilt; dennoch konstatiert sie, dass sie von ihrer Tätigkeit kaum leben könne. Bisweilen sei das Honorar öffentlicher Kultureinrichtungen respektive Förderungen so gering, dass die Befragte mit sechs Monaten Projektarbeit ihren Lebensunterhalt für nur zwei Monate absichern kann.

Den individuellen Marktrisiken begegnen viele Akteure, indem sie ihre Tätigkeiten diversifizieren und sich Marktnischen suchen. So entwickeln sie Projekte im öffentlichen und gemeinnützigen Kultursektor ebenso wie in der freien Wirtschaft. Sie erfüllen nicht unbedingt gängige Berufsbilder, sondern kreieren mit ihren Fähigkeiten Individualberufe. Eine Befragte erklärt etwa, sie sei „Produktionsdramaturgin“ - ein Profil, das die Bereiche Produktionsleitung und Dramaturgie in Theater und Oper verbindet.
Zudem übernehmen viele Akteure berufsferne „Brotjobs“, etwa als Immobilienmakler oder in Call-Centern. Diese bessern zwar das Einkommen auf, verhindern aber eine individuelle Weiterqualifizierung in dem eigentlichen Arbeitsfeld der Befragten. Sie tragen zu einer schleichenden Dequalifizierung bei, die nur durch entsprechende Mehrarbeit, einen höheren Zeitaufwand etc. abgefangen werden kann.

Nach Beobachtung der befragten Experten haben viele Kreative mentale Techniken entwickelt, um mit den vielfältigen Unsicherheiten wirtschaftlicher Natur, aber auch in Bezug auf erwerbsbiografische sowie private Planungsunsicherheiten, also mit dem umzugehen, was soziologisch als "prekär" eingestuft wird. Dazu gehöre, wirtschaftlich den „Ball flach zu halten“, z. B. sich in Wohn- oder Konsumfragen einzuschränken.

\section{INSTITUTIONELL-RECHTLICHE DIMENSION}

Alleinunternehmer in der Kreativwirtschaft genießen kaum sozialen, tarifpolitischen und rechtlichen Schutz. Ihre Arbeit wird nicht verbindlich geregelt, wie etwa bei Festangestellten oder den klassisch freien, verkammerten Berufen. Die Mitgliedschaft in Berufsverbänden ist freiwillig; diese sind nicht in korporatistische Strukturen eingebunden und verfügen nur über eine geringe Regulationsmacht - ihre Rolle beschränkt sich meist auf die interne Vermittlung von Preis- und Qualitätsstandards (Betzelt 2006). Auch die soziale Absicherung für Alter, Krankheit und Pflege ist nur ungenügend. Mit der Künstlersozialkasse (KSK) existiert zwar eine gesetzliche Mindestabsicherung für Künstler und Publizisten, sie beinhaltet aber keine Absicherung im Falle von Arbeitslosigkeit (Gottschall/Schnell 2000).

Mit der Zunahme eingesessener und neuer Kulturberufe sowie dem parallel verlaufenden Stellenabbau im öffentlichen Kulturbereich gibt es jedoch zunehmend mehr Kulturschaffende, die außerhalb dieses sozialen Sicherungssystems stehen (Haak 2005). Für Alleinunternehmer bleibt nur der private Versicherungs- und Vorsorgeschutz, der aus finanziellen Gründen meist nur unzureichend, oftmals gar nicht erfolgt.

Außerdem handelt es sich bei künstlerischen und publizistischen Tätigkeiten um offene Berufe ohne einheitlichen Ausbil- dungsstandard. So kann sich jeder etwa Fotodesigner nennen und suggeriert damit, Fotograf zu sein. Verschärfend kommt hinzu, dass die Akteure ihre Interessen individualisiert vertreten müssen, da ihre hoch individualisierten Erwerbsfelder weitgehend eine "gewerkschaftsfreie Zone" sind (Teipen 2006, S. 58). Obwohl sich mit dem von ver.di betriebenen Beratungsnetz "mediafon" eine bekannte Beratungsinstanz entwickelt hat, gilt für kreativwirtschaftliche Branchen, dass insbesondere die Alleinunternehmer auf sich selbst sowie auf in Eigenregie gebildete Netzwerke zurückgeworfen sind. ${ }^{3}$

Der unsicheren institutionell-rechtlichen Einbindung begegnen die meisten Akteure mit einer guten Portion Ignoranz und Beharrlichkeit. So berichten die Befragten, dass sie sich bei der Künstlersozialkasse (KSK) mitunter über Jahre hinweg beworben haben, bis der eigenschöpferische Anteil ihrer Arbeit anerkannt wurde.

Die fehlende institutionell-rechtliche Anerkennung wiegt für die Befragten oft schwerer als die unsichere finanzielle Lage. Sie fühlen sich in ihrer Tätigkeit weder gesellschaftlich wahrgenommen noch anerkannt. Ein Befragter, der in Eigeninitiative ein Medienfestival organisierte, erklärt: „Für meine Lebenssituation würde ich mir schon wünschen, dass es Möglichkeiten gibt, Ein-Mann-Unternehmen etwas mehr zu unterstützen, und die mir vor allem einen Status geben." Er erfährt das Leben jenseits der industriegesellschaftlich geprägten Normalbiografie als zweifelhaftes Privileg: „Ich würde mich immer als ProjectDirector, Kulturvermittler, Kurator und Regisseur vermitteln, aber meine Lebenssituation entspricht dem in keiner Weise“.

Für die Akteure ergibt sich somit eine widersprüchliche Situation: Sie verfügen über eine akademische Bildung und umfassende berufliche Qualifikationen, ihre soziale Lage spiegelt dies aber nicht wider. Vielmehr befinden sie sich subjektiv wie objektiv in einer sozialen Schwebesituation. Häufig sind die Befragten zeitweilig auf Hartz IV angewiesen. Die Befragten beschreiben diese Situation als ambivalent, weil sie damit zwar eine soziale Grund-

\footnotetext{
3 Mediafon ist ein gewerkschaftlicher Service für Soloselbstständige, der nach seinem Selbstverständnis eine praxisnahe und individuelle Beratung unter Kollegen bietet, aber keine wirkungsvolle regulatorische Kraft im sozialpolitischen Sinne besitzt (vgl. http://www.mediafon.net; 20.08.08).
} 
sicherung und sozialen Übergangsstatus erhalten, der ihnen allerdings nur eine prekäre Existenz bietet. Vor diesem Hintergrund wächst etwa die Mitgliedschaft in der KSK über den Stellenwert einer günstigen Kranken- und Rentenversicherung hinaus zu einer institutionellen Anerkennung und der Gewissheit, „dazu“ zu gehören.

\section{SOZIAL-KOMMUNIKATIVE DIMENSION}

In sozial-kommunikativer Hinsicht zeigen sich Widersprüche zu den herkömmlichen Prekaritätsdimensionen, denn die befragten Kreativen sind durchweg sozial sehr gut eingebunden und sowohl in berufsinterne als auch in berufsübergreifende Netzwerke integriert. Netzwerke sind die notwendige Bedingung, um Zugang zum jeweiligen Markt zu finden und sich darin zu behaupten. Dafür müssen die Akteure in den Netzwerken konstant an ihrer Marktposition arbeiten. Wie Haak/Schmidt (1999, S. 22) ausführen, sind die zentralen Steuerungsmedien hierfür Kommunikation, Vertrauen und der Ruf.

Der Aufbau und die Pflege der Netzwerke finden neben der eigentlichen Erwerbsarbeit statt und bedürfen zusätzlicher Ressourcen. Oftmals vermischen sich die Sphären „Arbeit" und „Privat", sodass sich Netzwerke herausbilden, in denen soziale und ökonomische Interaktionsbeziehungen nicht voneinander zu trennen sind. Dabei zeigt sich, dass eher die Struktur (also wen man kennt) als das Volumen (wie viele man kennt) des sozialen Kapitals darüber entscheidet, ob die Tätigkeit in der Kreativwirtschaft existenzsichernd ist. Denn von der strukturellen Ausgestaltung der Netzwerkbeziehungen und der Spezialisierung auf Marktsegmente hängt es ab, ob die Aufträge finanziell einträglich sind oder eine prekäre Existenz untermauern, wie etwa im Fall der Befragten, die auf öffentliche Förderung für ihre Projekte angewiesen sind.

Außerdem bedarf es eines spezifischen "State of Mind“, wie es ein Befragter pointierte. Dieser lässt sich als eine gekonnte Ausblendung oben erläuterter Rahmenbedingungen lesen, welche den Akteuren ermöglicht, sich als autonome Subjekte zu erleben, indem sie den Schritt ins Ungewisse hinein wagen und die Ausweitung von Unsicherheit offensiv aufgreifen (Manske 2006).
Zwar erfahren die Befragten die mit ihrer sozialen Lage verquickten Unsicherheiten als Zerreißprobe, die gleichwohl im subjektiven Erleben gemildert wird durch die Gewissheit, dass sie im sozioökonomischen Nahbereich als geteiltes Schicksal gilt. Auf der anderen Seite steht ein Gefühl von Handlungsautonomie, das eine Bewältigungsressource unter den prekären Bedingungen einer ökonomischen und institutionell-rechtlichen Knappheitssituation darstellt.

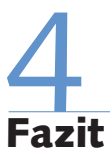

Am Beispiel der Kreativwirtschaft werden die konzeptionellen Grenzen der auf Industriearbeit fokussierten Prekaritätsdebatte deutlich. Denn insgesamt stellt die Kreativwirtschaft eher einen gesellschaftspolitischen und arbeitsmarkttheoretischen Sonderfall als ein potenzielles Pioniersegment künftiger Entwicklungen am Arbeitsmarkt dar. Denn die strukturellen Eigenheiten der Kreativwirtschaft, wie die projektbestimmte, kurzfristige Auftragsstruktur mit wechselnden Auftraggebern, sind nicht mit einem Normalarbeitsverhältnis zu vergleichen (Manske/Schnell 2009).

In Anlehnung an Dörres analytische Strukturdimensionen von Prekarität wurde gleichwohl gezeigt, dass eine prekäre Lebenslage für die Akteure in verschiedenen Dimensionen gleichzeitig auftritt. Die soziale Existenz von Alleinunternehmern ist fragil und verwundbar, weil sie Marktschwankungen ohne institutionalisierte Schutzmechanismen ausgesetzt sind und sich weder auf soziale Teilhaberechte noch auf ihre individuelle Daseinsvorsorge verlassen können. Alleinunternehmer der Kreativwirtschaft sind als radikal marktabhängig zu betrachten, da ihre soziale Existenz aus wohlfahrtsstaatlichen Arbeitsmarktprozessen herausgelöst ist und somit außerhalb erwerbsbezogener Sicherungssysteme sowie außerhalb traditioneller arbeitspolitischer und/oder ständischer Strukturierung der Erwerbsverhältnisse steht.

Vor allem in institutionell-rechtlicher Perspektive sind immer mehr Selbstständige in den Kulturberufen von einer sozialen Regulierung, die über die Grundsicherung hinausgeht, ausgeschlossen. Unsicherheiten beziehen sich in der Kreativwirtschaft daher erstens auf materielle Aspekte und zweitens auf institutionell-rechtliche Aspekte der Soziallage von Alleinunternehmern. Die Erwerbslage dieser Kreativen ist insoweit prekär, als sie eindeutig die herkömmlichen Standards von freiberuflichen Professionen als auch von abhängigen Beschäftigungsverhältnissen unterlaufen. Charakteristisch für die Akteure ist, dass sie eine hohe Bildung mit einem geringen Einkommen bei einer hohen sozialen und geringen institutionellen Integration verbinden. Bezieht man zudem die bislang ungebremste wirtschaftliche Dynamik und die Zuspitzung sozialer Ungleichheiten in der Kreativwirtschaft ein, dann zeigt sich, dass sich kreative Alleinunternehmer nicht nur mit Statusinkonsistenzen arrangieren müssen, sondern dass die wirtschaftliche Dynamik dieses "Zukunftsfeldes“ an vielen Akteuren vorbeigeht und dass die Entwicklung von Unsicherheitsbewältigungsstrategien eine unabdingbare Ressource in diesem Erwerbsfeld ist. Dabei erweist sich das soziale und kulturelle Kapital der Akteure als Bewältigungsressource der erfahrenen sozioökonomischen und soziopolitischen Unsicherheiten.

Die Befunde stützen somit neuere Untersuchungen, die für den Kulturbereich von einer Zunahme der Soloselbstständigkeit und „prekärer Selbstständigkeit“ auch in den „oberen Etagen der Arbeitsgesellschaft" sprechen (Schulze Buschoff 2006; Manske 2007). Mehr noch, die Analyse untermauert ein spezifisches Kennzeichen des aktuellen arbeitsgesellschaftlichen Strukturwandels: die Normalisierung, Ausbreitung und Verfestigung von Prekarität als strukturelles Phänomen (Manske 2008). So sind nicht existenzsichernde Einkommen, eine fehlende soziale Regulierung, überdurchschnittlich hohe Arbeitszeiten und eine wachsende Arbeitsmarktkonkurrenz für Kulturberufe mittlerweile auch im öffentlichen Kultursektor charakteristisch. Während jedoch für den Industriebereich strittig ist, wie weit die Destabilisierung des Normalarbeitsverhältnis reicht, war dieses im Kulturbereich schon immer die Ausnahme und kann deshalb kein normativer Fluchtpunkt sein (Lazzarato 2007).

Die Arbeitsmarktlage von kreativen Alleinunternehmern ist folglich nicht im Kontext des Normalarbeitsverhältnis zu deuten, da sie erstens im Kern den Künstlerarbeitsmärkten und den Erwerbsbedingungen von Künstler- und Kulturberufen folgt und in dieser historischen Genealogie 
betrachtet werden muss. Zweitens ist die Expansion des Arbeits- und Wirtschaftsfeldes Kreativwirtschaft mit einer Ausdifferenzierung seiner Erwerbsoptionen verknüpft, die sich ähnlich den Entwicklungen am Arbeitsmarkt allgemein in heterogener werdenden Beschäftigungsverhältnissen ausdrückt. Vor allem aber und drittens fügen sich Kreative nicht in das übliche Steckbild der Prekarier ein (Manske 2009).

Prekarisierung wird demnach insgesamt in zwei Formen sichtbar: zum einen in der Umwandlung gesicherter Arbeitsverhältnisse in ungesicherte. Diese werden von Castel (2000) allgemein und im deutschsprachigen Kontext speziell von Dörre umfangreich beschrieben und analysiert. Zum anderen äußert sich Prekarisierung in einer zweiten Form, die typisch für die hier beschriebenen Alleinunternehmer ist. Sie artikuliert sich in einer Normalisierung von Unsicherheit, der die Akteure mit unterschiedlichen Strategien begegnen und die sie mit differenzierten beruflichen Sinngehalten und Perspektiven verknüpfen. Ein wesentlicher Unterschied zu den Analysen zum Positionsverlust von Industriearbeitern liegt also in den subjektiven Verarbeitungsmustern. Während etwa für den Bereich der Leiharbeit von einem latenten Integrationsversprechen ausgegangen wird, welches Leiharbeiter zu einer enormen Leistungsverausgabung antreibe (Dörre 2005), gibt es in der Kreativwirtschaft subjektive Verarbeitungsmuster, die auf eine Normalisierung von Unsicherheit in zwei Dimensionen hindeuten.

Auf der synchronen Ebene zeigt sich, dass die Befragten ihre soziale Lage nicht als eine vorübergehende oder gar atypische Statuspassage empfinden, sondern als ein auf Dauer gestelltes Arbeitsverhältnis, das sie als charakteristisch für die Kreativwirtschaft als Arbeitsmarkt deuten. Mit einer zweiten, als diachron bezeichneten Ebene ist mit "Normalisierung von Unsicherheit" eine langfristige Perspektive gemeint. Denn eine industriegesellschaftlich geprägte Normalbiografie ist vom heutigen Erkenntnisstand gesehen und unter der Voraussetzung, dass sich die politische Regulierung der Kreativwirtschaft nicht fundamental verändert, für die übergroße Mehrheit der Kreativen ausgeschlossen. Ob es sich um eine „prekäre Stabilität“ (Vogel 2006, S. 86) handelt, die sich zwar irgendwie prekär, aber doch als halbwegs verlässlich darstellt, muss angesichts der präsentierten qualitativen Befunde zur wirtschaftlichen und sozialen Lage von Alleinunternehmern der Kreativwirtschaft angezweifelt werden. Vielmehr kann in Anlehnung an Serge Paugam (2009, S. 184) argumentiert werden, dass es sich im vorliegenden Fall um eine unsiche- re Integration in den Arbeitsmarkt handelt, in der ein instabiles Beschäftigungsverhältnis nicht mit unbefriedigender Arbeit einhergeht. Es ist indessen diese Besonderheit, welche die Kreativen zu besonders ,geduldigen Prekariern" macht. Prekarisierung auf hohem Niveau hat folglich eine Doppelbedeutung, die sich in einer Paarung von unstetem Beschäftigungsverhältnis und hoher arbeitsinhaltlicher Identifikation äußert und insofern auf neue Formen von Soziabilität verweist (Gill/Pratt 2008, S. 3).

Die präsentierten Befunde weisen somit auf die „feinen Unterschiede“ sozialer Gefährdungen und Risiken hin. Deshalb, so unser Fazit, ist die wirtschaftliche und soziale Lage von Alleinunternehmern der Kreativwirtschaft als prekär und radikal ungewiss einzustufen, aber nicht als atypisch. Wenn wir daher von Prekarisierung auf hohem Niveau sprechen, dann sind damit nicht nur eine beschäftigungspolitische Lage und ihre lebensweltlichen Implikationen gemeint, Vielmehr sprechen wir von einer Position im sozialen Raum, die einen spezifischen Brennpunkt des arbeitsgesellschaftlichen Restrukturierungsprozesses akzentuiert, der in der Prekaritätsdiskussion zu kurz kommt. 


\section{LITERATUR}

Betzelt, S. (2006): Flexible Wissensarbeit: AlleindienstleisterInnen zwischen Privileg und Prekarität, ZeS-Arbeitspapier 3-2006, Zentrum für Sozialpolitik, Universität Bremen

Castel, R. (2000): Die Metamorphosen der sozialen Frage. Eine Chronik der Lohnarbeit, Konstanz

Creative Industries Mapping Document (2001): Herausgegeben vom Ministerium für Kultur, Medien und Sport (DCMS) der britischen Regierung. http://www.culture.gov.uk/global/publications/archive_2001/ci_ mapping_doc_2001.htm (20.08.2007)

Dörre, K. (2005): Prekarität - Eine arbeitspolitische Herausforderung, in: WSI-Mitteilungen 5, S. 250-258

Florida, R. (2002): The Rise of the Creative Class, New York Geppert, K./Mundelius, M. (2007): Berlin als Standort der Kreativwirtschaft immer bedeutender, DIW Wochenbericht 31, S. 485-491

Gill, R./Pratt, A. C. (2008): In the Social factory? Immaterial Labour, Precariousness and Cultural Work, in: Theory, Culture and Society 7-8, S. 1-30

Gottschall, K. (1999): Freie Mitarbeit im Journalismus. Zur Entwicklung von Erwerbsformen zwischen selbständiger und abhängiger Beschäftigung, in: Kölner Zeitschrift für Soziologie und Sozialpsychologie 4, S. 635-654

Gottschall, K./Schnell, C. (2000): Alleindienstleister in Kulturberufen Zwischen neuer Selbständigkeit und alten Abhängigkeiten, in: WSI-Mitteilungen 12, S. 804-810

Haak, C. (2005): Künstler zwischen selbstständiger und abhängiger Erwerbsarbeit, Wissenschaftszentrum Berlin für Sozialforschung, Discussion Paper SP I 2005-107

Haak, C./Schmid, G. (1999): Arbeitsmärkte für Künstler und Publizisten Modelle einer zukünftigen Arbeitswelt? Wissenschaftszentrum Berlin für Sozialforschung, Discussion Paper P99-506

Henninger, A. (2004): Free Lancer in den Neuen Medien: Jenseits standardisierter Muster von Arbeit und Leben?, in: Kahlert, H./Kajatin, C. (Hrsg.): Arbeit und Vernetzung im Informationszeitalter. Wie neue Technologien die Geschlechterverhältnisse verändern, Frankfurt, M./New York, S. 143-166

Keller, B./Seifert, H. (2006): Atypische Beschäftigungsverhältnisse: Flexibilität, soziale Sicherheit und Prekarität, in: WSI-Mitteilungen 5, S. 235240

Kultur in Deutschland (2007): Abschlussbericht der Enquete-Kommission

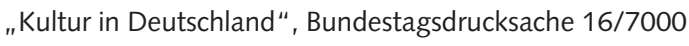

Lange, B. (2007): Die Räume der Kreativszenen. Culturepreneurs und ihre Orte in Berlin, Bielefeld

Lazzarato, M. (2007): Die Missgeschicke der Künstlerkritik und der kulturellen Beschäftigung; in: Raunig, G./ Wuggenig, U. (Hrsg.): Kritik der Kreativität, Wien, S.190-206

Leicht, R./Philipp, R. (2005): Die wachsende Bedeutung von Ein-Personen-Unternehmen in Deutschland: Wo und unter welchen Bedingungen arbeiten Selbständige zunehmend alleine? in: Welter, F. (Hrsg.): Dynamik im Unternehmenssektor: Theorie, Empirie und Politik, Berlin, S. 131-154 Mandel, B. (2007): Die neuen Kulturunternehmer. Ihre Motive, Visionen und Erfolgsstrategien, Bielefeld
Manske, A. (2006): Die Stellung halten. Marktstrategien und Positionskämpfe in Berlins Internetindustrie, in: Soziale Welt 2, S. 159-176 Manske, A. (2007): Prekarisierung auf hohem Niveau. Eine Feldstudie über Allein-Unternehmer in der IT-Branche, München und Mering Manske, A. (2008): Das Ende der Eindeutigkeiten? Die Prekarisierung der Arbeitsgesellschaft aus genderpolitischer Perspektive; in: Geissel, B./ Manske, A. (Hrsg.): Kritische Vernunft für demokratische Transformationen. Festschrift für Christine Kulke, Opladen, S. 140-168 Manske, A. (2009): Unsicherheit und kreative Arbeit - Stellungskämpfe von Soloselbständigen in der Kulturwirtschaft; in: Castel, R./ Dörre, K. (Hrsg.): Prekarität, Abstieg, Ausgrenzung. Die soziale Frage am Beginn des 21. Jahrhunderts, Frankfurt M./New York, S. 283-296

Manske, A./Merkel, J. (2008): Kreative in Berlin. Eine Untersuchung zum Thema "GeisteswissenschaftlerInnen in der Kultur- und Kreativwirtschaft" "Wissenschaftszentrum Berlin für Sozialforschung, Discussion Paper SP III 2008-401

Manske, A./Schnell, C. (2009): Kultur- und Kreativarbeit. Deutungen und Befunde im Kontext des Strukturwandels der Arbeitsgesellschaft; in: Böhle, F. / Voß, G. G./ Wachtler, G. (Hrsg.): Handbuch zur Arbeitssoziologie, Frankfurt. M./New York (i. E.)

Mayer-Ahuja, N. (2003): Wieder dienen lernen? Vom westdeutschen "Normalarbeitsverhältnis“ zu prekärer Beschäftigung seit 1973, Berlin Merkel, J. (2009): Kreativquartiere. Urbane Milieus zwischen Inspiration und Prekarität, Berlin

Paugam, S. (2009): Die Herausforderung der organischen Solidarität durch die Prekarisierung von Arbeit und Beschäftigung, in: Castel, R./ Dörre, K. (Hrsg.): Prekarität, Abstieg, Ausgrenzung. Die soziale Frage am Beginn des 21. Jahrhunderts, Frankfurt M./New York, S. 175-196

Schnell, C. (2008). Selbständige zwischen Professionalität, Prekarität und kollegialen Allianzen. Befunde aus dem Feld der Kulturberufe, in: Kock, K. (Hrsg.): Preis der Freiheit. Solo-Selbständige zwischen Vermarktung, Professionalisierung und Solidarisierung, Sozialforschungsstelle Dortmund, Beiträge aus der Forschung, S. 8-17

Schulze Buschoff, K. (2006): Selbständige Erwerbsarbeit und soziales Risikomanagement - ein deutsch-britischer Vergleich, in: Zeitschrift für Sozialreform 4, S. 521-546

Söndermann, M. (2007): Kulturwirtschaft und Creative Industries 2007. Allgemeine empirische Trends und mit Schwerpunkt: Kleine Kulturwirtschaft. Im Auftrag der Bundestagsfraktion Bündnis 90 /Die Grünen, Juni Teipen, C. (2006): Arbeit und Beschäftigung in kreativen Industrien - Entwicklungen in der Computerspielindustrie in Deutschland, Schweden und Polen, Wissenschaftszentrum Berlin für Sozialforschung, Discussion Paper SP III 2006-301

Vogel, B. (2006): Sicher - Prekär; in: Lessenich, S./Nullmeier, F. (Hrsg.): Deutschland - eine gespaltene Gesellschaft, Bonn, S. 73-91

Voß, G. G. (2007): Subjektivierung von Arbeit und Arbeitskraft. Das Beispiel Gender, in: Aulenbacher, B./Funder, M./Jacobsen, H./Völker, S. (Hrsg.): Arbeit und Geschlecht im Umbruch der modernen Gesellschaft, Wiesbaden, S. 97-113 